\title{
Probing the diffuse baryon distribution with the lensing-tSZ cross-correlation
}

\section{Yin-Zhe Ma, ${ }^{a, b}$ Ludovic Van Waerbeke, ${ }^{b}$ Gary Hinshaw, ${ }^{b, c}$ Alireza Hojjati, ${ }^{b, d}$ Douglas Scott, ${ }^{b}$ Joe Zuntz ${ }^{a}$}

\author{
${ }^{a}$ Jodrell Bank Centre for Astrophysics, School of Physics and Astronomy, The University of Manch- \\ ester, Oxford Road, Manchester, UK. M13 9PL \\ ${ }^{b}$ Department of Physics and Astronomy, University of British Columbia, 6224 Agricultural Road, \\ Vancouver, V6T 1Z1, BC Canada. \\ ${ }^{c}$ Canada Research Chair in Observational Cosmology \\ ${ }^{d}$ Simon Fraser University, 8888 University Drive Burnaby, B.C. Canada V5A 1S6 \\ E-mail: mayinzhe@manchester.ac.uk
}

\begin{abstract}
Approximately half of the Universe's baryons are in a form that has been hard to detect directly. However, the missing component can be traced through the cross-correlation of the thermal Sunyaev-Zeldovich (tSZ) effect with weak gravitational lensing. We build a model for this correlation and use it to constrain the extended baryon component, employing data from the Canada France Hawaii Lensing Survey and the Planck satellite. The measured correlation function is consistent with an isothermal $\beta$-model for the halo gas pressure profile, and the 1- and 2-halo terms are both detected at the $4 \sigma$ level. In addition, we measure the hydrostatic mass bias $(1-b)=0.79_{-0.10}^{+0.07}$, which is consistent with numerical simulation results and the constraints from X-ray observations. The effective temperature of the gas is found to be in the range $\left(7 \times 10^{5}-3 \times 10^{8}\right) \mathrm{K}$, with approximately $50 \%$ of the baryons appearing to lie beyond the virial radius of the halos, consistent with current expectations for the warm-hot intergalactic medium.
\end{abstract}

Keywords: Cosmology: large scale structure, observations, theory, Sunyaev-Zeldovich effect, gravitational lensing

ArXiv ePrint: 1404.4808 


\section{Contents}

1 Introduction 1

2 Lensing-tSZ cross-correlation data 2

$3 \kappa$ - $y$ correlation function in the halo model 2

4 Pressure profile 4

4.1 Models for gas pressure $\quad 4$

4.2 Hydrostatic mass bias 5

4.3 WMAP and Planck cosmological parameters 6

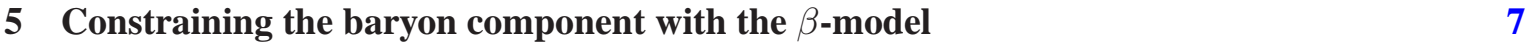

6 Discussion and Conclusions 9

\section{Introduction}

The general processes driving structure formation, from the sizes of galaxies to the largest scales observable, are reasonably well understood, though many details are still unclear. Knowledge of the distribution of baryonic and dark matter in galaxies, groups, and clusters of galaxies is essential for understanding how they form and evolve, including complex processes such as down-sizing and star-formation quenching $[1,2]$. However, stellar mass accounts for only $\sim 10 \%$ of all the baryons in the Universe; the other $90 \%$ resides in a diffuse component, a large fraction of which is thought to reside in low mass halos [3]. A complete picture of structure formation requires a full census of baryons in the Universe. Baryons are more dissipative than dark matter, and hence naturally populate the centres of halos, but feedback processes play a fundamental role in recycling baryons back to a diffuse form. Thus, accounting for the extended baryon distribution is necessary to understand the physical processes governing structure formation, including star formation and feedback.

Historically, the diffuse component is observed via its X-ray emission or through the thermal Sunyaev-Zeldovich effect (tSZ, i.e., inverse Compton scattering [4]), but the sensitivity of current instruments limits such observations to the most massive, densest, and hottest gas environments. To date, only about half of the known baryon component has been directly observed at redshifts less than $z \simeq 2[3,5]$; the remaining baryons are thought to be too cold to be detected with X-rays or the SZ effect, and too warm to be detected in the UV. Numerical simulations suggest that the "missing" baryons might be in a warm, low-density plasma $\left(\sim 10^{5}-10^{7} \mathrm{~K}\right)$ correlated with large structures and filaments [6].

One possible way of observing these baryons is by cross-correlating with another cosmic field. Gravitational lensing by large-scale structure provides an unbiased tracer of the matter distribution that can be used for this purpose. Van Waerbeke, Hinshaw \& Murray [7] found a significant correlation between the Canada France Hawaii Lensing Survey (CFHTLenS) mass map and tSZ maps obtained from Planck satellite data. This signal was consistent with warm baryonic gas tracing largescale structure, with an amplitude $\bar{n}_{\mathrm{e}} T_{\mathrm{e}} b_{\text {gas }} \simeq 0.201 \mathrm{keV} \mathrm{m}^{-3}$ at redshift $z=0$. This suggests that if the bias $b_{\text {gas }} \simeq 6$ and $\bar{n}_{\mathrm{e}}=0.25 \mathrm{~m}^{-3}$ (the cosmic baryon abundance), then it is in line with the missing baryons being at $T_{\mathrm{e}} \simeq 10^{6} \mathrm{~K}$. 
The model adopted for the warm gas in Ref. [7] was simplistic and did not capture some of the essential physical properties. It assumed: (i) that the temperature and density of the gas are independent of the underlying halo mass and redshift; and (ii) that the bias of gas pressure relative to dark matter follows $b_{\text {gas }} \propto a$, where $a$ is the cosmic scale factor, independent of halo mass and redshift. Moreover the formalism used could not account for gas lying outside single halos, thus it was incapable of tracking the "missing baryons" that are thought to reside outside the cluster virial radius. Here we attempt to provide a realistic description of the baryon distribution within the framework of the "halo model" [8]. By interpreting the cross-correlation between tSZ and lensing we investigate the consequences for the warm baryonic component.

Except for Fig. 2, we use best-fit cosmological parameters obtained from the Planck satellite [9] throughout the paper, i.e., $\left\{\Omega_{\mathrm{m}}, \Omega_{\mathrm{b}}, \Omega_{\Lambda}, \sigma_{8}, n_{\mathrm{s}}, h\right\}=\{0.3175,0.0490,0.6825,0.834,0.9624$, $0.6711\}$.

\section{Lensing-tSZ cross-correlation data}

The tSZ effect is produced by inverse-Compton scattering of cosmic microwave background (CMB) photons off electrons in the hot intra-cluster gas. At frequency $\nu$, this induces a temperature anisotropy along the line-of-sight characterized by the Compton $y$-parameter,

$$
\frac{\Delta T}{T_{0}}=y S_{\mathrm{SZ}}(x), y=\int n_{\mathrm{e}} \sigma_{\mathrm{T}} \frac{k_{\mathrm{B}} T_{\mathrm{e}}}{m_{\mathrm{e}} c^{2}} \mathrm{~d} l,
$$

where $S_{\mathrm{SZ}}(x)=x \operatorname{coth}(x / 2)-4$ gives the tSZ spectral dependence [4]. Here, $x=h \nu / k_{\mathrm{B}} T_{0}, n_{\mathrm{e}}$ is the electron density, $\sigma_{\mathrm{T}}$ is the Thomson cross-section, $T_{\mathrm{e}}$ is the electron temperature, and $T_{0}$ is the present-day CMB temperature.

For this analysis, we use the cross-correlation data described in Ref. [7]. The gravitational lensing mass map, $\kappa$, is based on CFHTLenS data [10-14] and covers $154 \mathrm{deg}^{2}$ in four separate patches. The tSZ $y$ maps are obtained from a linear combination of the four Planck channel maps at $100,143,217$, and $353 \mathrm{GHz}$ [15]. The $\kappa$ and $y$ maps are smoothed by a Gaussian beam with FWHM of 10 arcmin and 9.5 arcmin, respectively. Several different $y$ maps were produced in order to test for contamination of the SZ signal by thermal dust and $\mathrm{CO}$ line emission. These maps, labeled B-H in Ref. [7], were constructed using different channel combinations, which would be expected to have very different levels of signal contamination. As already noted, the expected SZ contamination levels range over more than a factor of 6 across this set, while the measured cross-correlation signal varies by less than $10 \%$. In this paper, we have adopted SZ map D as our best estimate of the foregroundreduced $y$ map, because it projects out dust assuming $\beta_{\text {dust }}=1.8$, similar to the recent findings by the Planck collaboration [16]. However, as noted in Ref. [7] using different spectral indices for the dust de-projection has only a $10 \%$ effect on our signal and does not affect our conclusions.

\section{$3 \kappa-y$ correlation function in the halo model}

The lensing-tSZ cross-correlation power spectrum is the sum of two terms, $C_{\ell}^{\kappa y}=C_{\ell}^{\kappa y, 1 \mathrm{~h}}+C_{\ell}^{\kappa y, 2 \mathrm{~h}}$, where " $1 \mathrm{~h}$ " and " $2 \mathrm{~h}$ " refer to the 1- and 2-halo terms, respectively. The 1-halo term, the Poissonian contribution, is given by [8]

$$
C_{\ell}^{\kappa y, 1 \mathrm{~h}}=\int_{0}^{z_{\max }} \mathrm{d} z \frac{\mathrm{d} V}{\mathrm{~d} z \mathrm{~d} \Omega} \int_{M_{\min }}^{M_{\max }} \mathrm{d} M \frac{\mathrm{d} n}{\mathrm{~d} M} y_{\ell}(M, z) \kappa_{\ell}(M, z),
$$



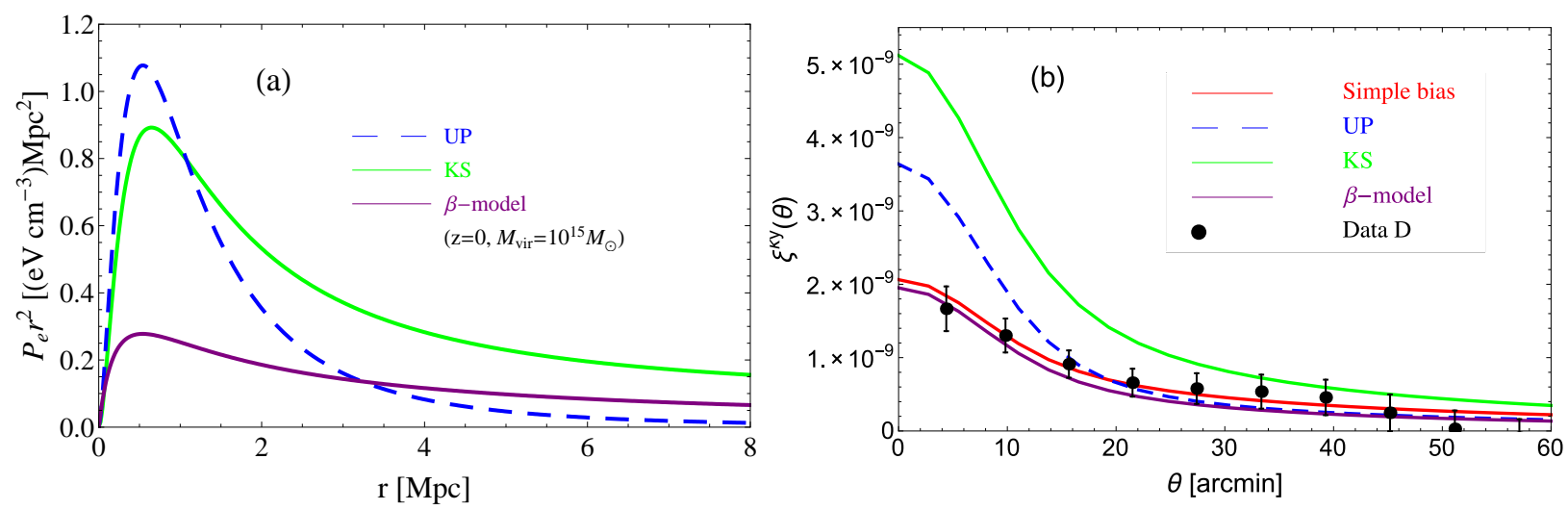

Figure 1. Models for: (a) the radial pressure profile within a halo; and (b) the $\kappa-y$ correlation function derived from the halo model. The pressure profiles considered are: red, the simple bias model, with $b_{\text {gas }} T_{\mathrm{e}} n_{\mathrm{e}}=$ $0.201 \mathrm{keV} \mathrm{m}^{-3}$ [7]; blue dashed, the universal pressure (UP) profile [24]; green, the Komatsu-Seljak (KS) profile [23]; and purple, the isothermal $\beta$-model profile [26]. The data points in panel (b) show the correlation function specifically for tSZ data set " $D$ ".
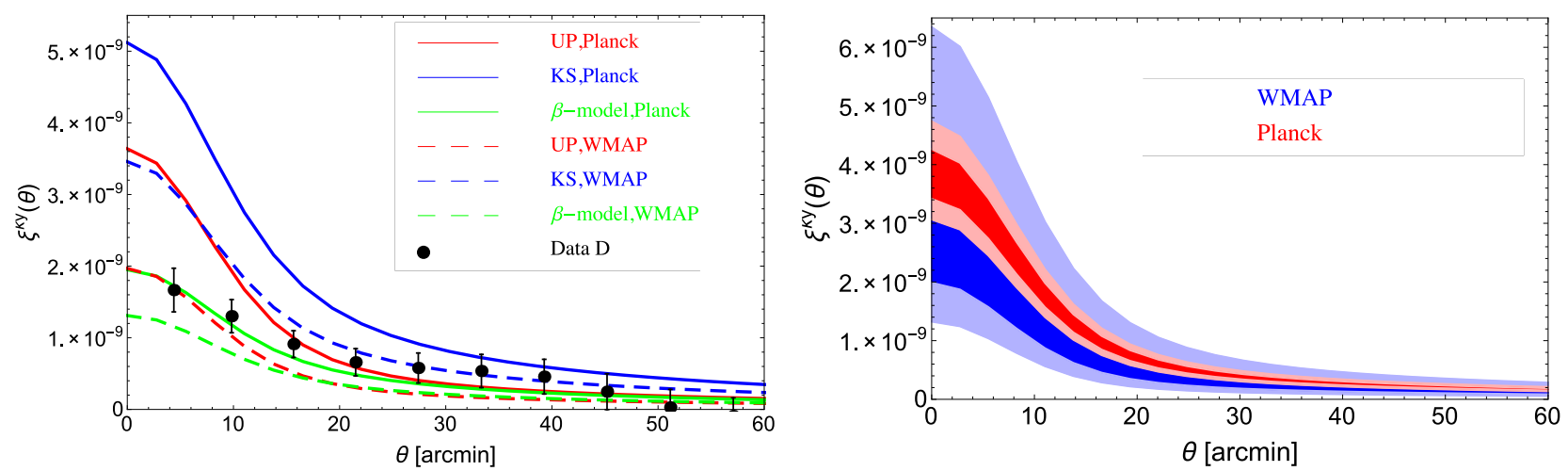

Figure 2. Left-Comparison of the model predictions using best-fit 7-year WMAP and Planck 2013 cosmological parameters. The values are $\left\{\Omega_{\mathrm{m}}, \Omega_{\mathrm{b}}, \Omega_{\Lambda}, \sigma_{8}, n_{\mathrm{s}}, h\right\}=\{0.272,0.0455,0.728,0.81,0.967,0.704\}$ for WMAP and $\{0.3175,0.0490,0.6825,0.834,0.9624,0.6711\}$ for Planck. Right- the same comparison for the UP model, but sampling the posterior distribution of cosmological parameter space. The deep (shallow) blue and red regions are for WMAP and Planck 68\% (95\%) confidence levels, respectively.

where $\mathrm{d} V /(\mathrm{d} z \mathrm{~d} \Omega)=c \chi^{2} / H(z)$ is the comoving volume per unit redshift and solid angle, with $\chi(z)$ the comoving distance to redshift $z$ (in the best-fit Planck cosmology [9]). The quantity $\mathrm{d} n / \mathrm{d} M$ is the halo mass function, taken here to be the Sheth-Tormen form [17]. The multipole functions $y_{\ell}$ and $\kappa_{\ell}$ are related to the halo gas and mass profiles, respectively, as now described.

The quantity $\kappa_{\ell}(M, z)$ is the Fourier transform of the convergence profile of a single halo of mass $M$ and redshift $z$ :

$$
\kappa_{\ell}=\frac{W^{\kappa}(z)}{\chi^{2}(z)} \frac{1}{\bar{\rho}_{\mathrm{m}}} \int_{0}^{r_{\mathrm{vir}}} \mathrm{d} r\left(4 \pi r^{2}\right) \frac{\sin (\ell r / \chi)}{\ell r / \chi} \rho(r ; M, z) .
$$

Here $\bar{\rho}_{\mathrm{m}}$ is the comoving matter density, $\rho(r ; M, z)$ is the matter halo profile, taken here to be the Navarro-Frenk-White (NFW) form [18], and $W^{\kappa}$ is the lensing kernel, which is given in Eq. (1) and plotted in figure 1 of Ref. [7]. For the CFHTLenS data, the kernel peaks at $z \simeq 0.37$; we have verified that Eq. (3.1) has converged at $z_{\max }=3.0$ and adopt this redshift cutoff. For the integral over mass 
we have verified that the integral has converged at a lower limit of $10^{12} \mathrm{M}_{\odot}$ and an upper limit of $10^{16} \mathrm{M}_{\odot}$ and thus adopt this mass range.

The quantity $y_{\ell}(M, z)$ in Eq. (3.1) is the 2-d Fourier transform of the projected gas pressure profile of a single halo of mass $M$ and redshift $z$ [19]:

$$
y_{\ell}=\frac{4 \pi r_{\mathrm{s}}}{\ell_{\mathrm{s}}^{2}} \frac{\sigma_{\mathrm{T}}}{m_{\mathrm{e}} c^{2}} \int \mathrm{d} x x^{2} \frac{\sin \left(\ell x / \ell_{s}\right)}{\ell x / \ell_{s}} P_{\mathrm{e}}(x ; M, z) .
$$

Here $x=a(z) r / r_{\mathrm{s}}, \ell_{\mathrm{s}}=a \chi / r_{\mathrm{s}}, r_{\mathrm{s}}$ is the scale radius of the 3 -d pressure profile, and $P_{\mathrm{e}}$ is the electron pressure. The ratio $r_{\mathrm{vir}} / r_{\mathrm{s}}$ is called concentration parameter, which we take to be [20]

$$
c=\frac{5.72}{(1+z)^{0.71}}\left(\frac{M_{\mathrm{vir}}}{10^{14} h^{-1} \mathrm{M}_{\odot}}\right)^{-0.081} .
$$

This is based on the assumption that the ratio of $r_{\mathrm{vir}} / r_{\mathrm{s}}$ for the gas profile follows the same ratio as for the NFW (dark matter) profile. We have verified that the integral in Eq. (3.3) has converged by $r=5 r_{\text {vir }}$ and adopt this as an upper cutoff radius.

The 2-halo term (to add to Eq. 3.1) is given by

$$
\begin{aligned}
C_{\ell}^{\kappa y, 2 \mathrm{~h}} & =\int_{0}^{z_{\max }} \mathrm{d} z \frac{\mathrm{d} V}{\mathrm{~d} z \mathrm{~d} \Omega} P_{\mathrm{m}}^{\operatorname{lin}}(k=\ell / \chi, z) \\
& \times\left[\int_{M_{\min }}^{M_{\max }} \mathrm{d} M \frac{\mathrm{d} n}{\mathrm{~d} M} b(M, z) \kappa_{\ell}(M, z)\right]\left[\int_{M_{\min }}^{M_{\max }} \mathrm{d} M \frac{\mathrm{d} n}{\mathrm{~d} M} b(M, z) y_{\ell}(M, z)\right],
\end{aligned}
$$

where $P_{\mathrm{m}}^{\operatorname{lin}}(k, z)$ is the 3 -d linear matter power spectrum at redshift $z$, which we obtained from the code CAMB [21], with the best-fit parameters from Planck [9]. Here $b(M, z)$ is the gravitational clustering bias function (from Ref. [22]). In order to compare the halo model to the cross-correlation data, $\xi^{\kappa y}(\theta)$, we Legendre transform $C_{\ell}^{\kappa y}$ into real space.

\section{Pressure profile}

\subsection{Models for gas pressure}

We consider three different gas models in this study: the Komatsu-Seljak (KS) profile [23]; the universal pressure (UP) profile [24]; and the isothermal $\beta$-model [25-28]. For the KS model, we use equations (D4)-(D13) in [29] to implement the pressure profile. For the UP model, the pressure is given by

$$
P\left(x \equiv r / R_{500}\right)=1.65 \times 10^{-3} E(z)^{\frac{8}{3}}\left(\frac{M_{500}}{3 \times 10^{14} h_{70}^{-1} \mathrm{M}_{\odot}}\right)^{\frac{2}{3}+\alpha_{\mathrm{p}}} \mathbb{P}(x) h_{70}^{2}\left[\mathrm{keV} \mathrm{cm}^{-3}\right],
$$

where $h_{70}=(h / 0.7), \alpha_{\mathrm{p}}=0.12$, and $M_{500}=(4 \pi / 3) 500 \rho_{\mathrm{c}}(z) R_{500}^{3}$, i.e., the total mass within the radius where the total density contrast is 500 . Here $\mathbb{P}(x)$ is the generalized NFW model [24]

$$
\mathbb{P}(x)=\frac{P_{0}}{\left(c_{500} x\right)^{\gamma}\left[1+\left(c_{500} x\right)^{\alpha}\right]^{(\beta-\gamma) / \alpha}},
$$

where $P_{0}$ is the overall magnitude of the pressure profile, and $c_{500}, \gamma, \alpha$, and $\beta$ determine the slope of the profile. We use the parameter set $\left\{P_{0}, c_{500}, \alpha, \beta, \gamma\right\}=\{6.41,1.81,1.33,4.13,0.31\}$, which is obtained as the best-fit values of 62 nearby massive clusters [30]. 

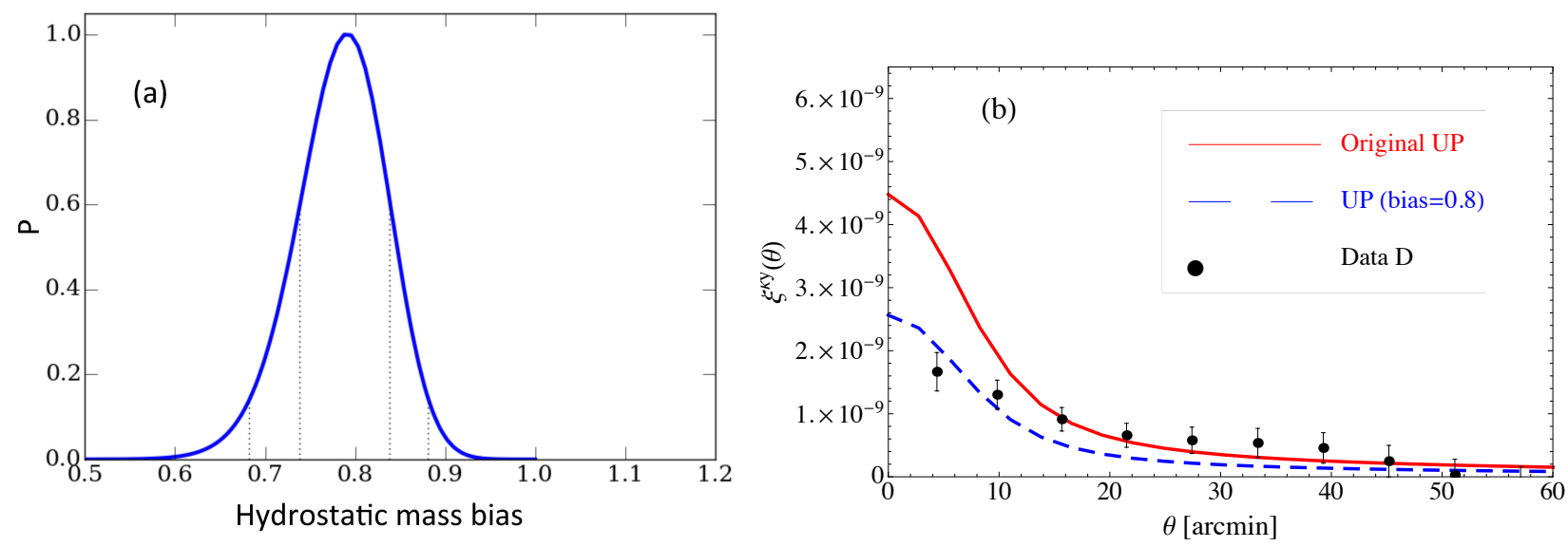

Figure 3. Left-marginalized likelihood function for the hydrostatic mass bias $1-b$ factor for data set "D" and the UP model. Right- comparison between the original UP model and a UP model with $1-b=0.8$.

For the isothermal $\beta$-model,

$$
n_{\mathrm{e}}(r)=n_{\mathrm{e} 0}\left[1+\left(\frac{r}{r_{\mathrm{s}}}\right)^{2}\right]^{-3 \beta / 2},
$$

and we use $\beta=0.86$, which is consistent with the fits to the X-ray surface brightness [27] and with fits to 15 stacked SZ clusters from South Pole Telescope data [28]. The quantity $r_{\mathrm{s}}=r_{\mathrm{vir}} / c$ is the scale radius that we use in Eq. (3.4), derived by assuming that the underlying dark matter distribution follows the NFW profile. For the central density, we fix the normalization with $4 \pi \int_{0}^{r_{\mathrm{vir}}} n_{\mathrm{e}}(r) r^{2} \mathrm{~d} r=$ $N_{\mathrm{e}}$, where $N_{\mathrm{e}}=\left(1+f_{\mathrm{H}}\right) M_{\text {vir }} f_{\text {gas }} /\left(2 m_{\mathrm{p}}\right)$ [26]. Here $f_{\mathrm{H}}=0.76$ is the hydrogen mass fraction, and $f_{\text {gas }}=\Omega_{\mathrm{b}} / \Omega_{\mathrm{m}}$ is the baryonic gas fraction of the Universe. We use equation (14) in Ref. [26] as the temperature in the $\beta$-model, where this equation was calibrated against 24 hydrodynamic cluster simulations [31]. Fig. 1a shows the pressure profiles for each model in a halo of mass $M_{\text {vir }}=$ $10^{15} \mathrm{M}_{\odot}$ at $z=0$. Note that the amplitudes of the KS and UP profiles are much higher than that of the $\beta$-model, the latter being fairly consistent with the simple bias approach from Ref. [7].

\subsection{Hydrostatic mass bias}

Figure $1 \mathrm{~b}$ (Fig. 6a) shows the predicted correlation functions (angular power spectrum), $\xi^{\kappa y}(\theta)\left(C_{\ell}^{\kappa y}\right)$, for each of the pressure profiles described above. Also shown is the measured correlation function using data set "D" from Ref. [7]. It is clear that the KS and UP models predict too much power at small scales, while the isothermal $\beta$-model lies reasonably close to the data at all scales. Note that once the cosmological parameters and gas model parameters have been chosen, a given gas model amplitude and profile has no further freedom to be adjusted.

In Fig. 3b, we plot the UP profile (as a red solid line), which predicts too much power on small angular scales compared to the data. In comparison, we plot $M_{\mathrm{obs}, 500}=(1-b) M_{\text {true,500 }}$ (as a dashed blue line), where $1-b=0.8$ is the hydrostatic mass bias between observed and true halo masses. One can see that the total amplitude is lowered, and the small angular scales fit better to the data, while on larger angular scales the prediction is still lower than the data. We run an MCMC chain to constrain this $1-b$ bias factor, and we obtained the likelihood as shown in Fig. 3a. The best-fit value is $(1-b)=0.79_{-0.1}^{+0.07}$ (at $\left.95 \% \mathrm{CL}\right)$. 


\begin{tabular}{ccccc}
\hline & Observation/Simulation & Quantity & Value (68\% CL) & Reference \\
\hline \multirow{5}{*}{ Data } & WtG & $1-b$ & $0.688 \pm 0.072$ & von der Linden et al. 2014 [32] \\
\cline { 2 - 5 } & CCCP & $1-b$ & $0.76 \pm 0.11$ & Hoekstra et al. 2015 [33] \\
\cline { 2 - 5 } & 400d survey & $b$ & $\simeq 20 \%$ & Israel et al. 2014 [34] \\
\cline { 2 - 5 } & X-ray \& WL & $1-b$ & $0.66_{-0.12}^{+0.07}$ & Simet et al. [35] \\
\cline { 2 - 5 } & CMB lensing & $1 /(1-b)$ & $0.99 \pm 0.19$ & Planck 2015 results XXIV [36] \\
\cline { 2 - 5 } & tSZ-CMB Lensing & $1-b$ & $1.06_{-0.14}^{+0.11}$ & Hill \& Spergel [37] \\
\cline { 2 - 5 } & CMB+SZ & $1-b$ & $0.58 \pm 0.04$ & Planck 2015 results XXIV [36] \\
\hline \multirow{4}{*}{ Simu- } & tSZ-Lensing correlation & $1-b$ & $0.79_{-0.10}^{+0.07}$ & This work \\
\cline { 2 - 5 } lations & Hydro-simulation & $b$ & $10 \%-20 \%$ & Shaw et al. 2010 [38] \\
\cline { 2 - 5 } & TreePM/SPH GADGET-3 & $b$ & $\sim 25 \%$ & Raisa et al. 2012 [39] \\
\cline { 2 - 5 } & Eulerian cluster & $b$ & $10 \%-20 \%$ & Nagai et al. 2007 [40] \\
\cline { 2 - 5 } & N-body/SPH & $b$ & $10 \%-15 \%$ & Piffaretti \& Valdarnini 2008 [41] \\
\cline { 2 - 5 } & N-body/SPH & $b$ & $5 \%-20 \%$ & Meneghetti et al. 2010 [42] \\
\hline
\end{tabular}

Table 1. Comparison between measurements of hydrostatic bias from different samples of real data and simulations. Here "WtG" is the Weighing the Giants project [32], "CCCP" stands for Canadian Cluster Comparison Project [33], while "400d" stands for the $400 \mathrm{deg}^{2}$ Galaxy Cluster Survey Weak Lensing program, which claims that their studies favour a small WL-X-ray mass bias, consistent with both vanishing bias and $20 \%$ bias [34]. The "tSZ-CMB Lensing" row refers to the cross-correlation between the thermal SZ map and the CMB Lensing map of Planck, and fitting the $(1-b)$ factor while fixing all other cosmological parameters with $W M A P 9$ values [37]. The "CMB+SZ" case is not a direct estimate of $1-b$, but gives the value required in order to reconcile the tension between CMB and SZ determinations on the $\sigma_{8}-\Omega_{\mathrm{m}}$ constraint.

In Table 1, we make a comparison between measurements of hydrostatic mass bias from real data and simulations. The simulations are listed in the second half of the table. One can see that the simulations consistently prefer a value of $b$ around $10-20 \%$. For measurement from real data, Ref. [32] compares the Planck cluster mass with the weak lensing mass from the WtG project for 22 massive clusters, and find that $(1-b)=0.688 \pm 0.072$, while Ref. [33] uses the same method for 50 clusters and finds a higher value. In addition, by simulating Planck observations, Ref. [43] proposes the method of comparing CMB-measured mass to X-ray-measured mass, thus in Ref. [36], by using this method, the Planck collaboration finds the value $1 /(1-b)=0.99 \pm 0.19$, which lies towards the higher end of possible bias values. Finally, in order to reconcile the apparent tension between the cosmological parameter (in particular $\sigma_{8}$ and $\Omega_{\mathrm{m}}$ ) between the CMB anisotropy measurements and SZ number counts, Ref. [36] finds that the value of $(1-b)$ needs to be as low as 0.58 ; this is clearly much lower than the simulation results and individual measurements.

Therefore, we conclude that our measurement of hydrostatic mass bias, i.e., the $(1-b)$ value, is consistent with previous simulation results, and measurements from the CCCP and 400d surveys. However, the value we found is slightly higher than the value found in the $\mathrm{WtG}$ project as well as the value necessary to reconcile the tension between $\mathrm{CMB}$ and $\mathrm{SZ}$ cosmological constraints, and slightly lower than the CMB lensing results; however, all are consistent within $2 \sigma$.

\subsection{WMAP and Planck cosmological parameters}

In the left panel of Fig. 2, we plot the predictions of $\xi^{\kappa y}(\theta)$ for three gas models by using the best-fit cosmological parameters from the 7-year Wilkinson Microwave Anisotropy Probe (i.e., WMAP) [29] and Planck 2013 results [9]. One can see that, since the WMAP-7 data prefer smaller values of $\sigma_{8}$ and $\Omega_{\mathrm{m}}$ than Planck, the central value of the correlation function drops somewhat for each different model. For the KS, UP, and $\beta$ models, the central values of the correlation function drop by about 


\begin{tabular}{cccc}
\hline Data set & 2-halo only & 1-halo only & No correlation \\
\hline B & $4.8 \times 10^{-4}$ & $6.7 \times 10^{-5}$ & $4.5 \times 10^{-11}$ \\
C & $1.9 \times 10^{-5}$ & $2.0 \times 10^{-4}$ & $1.3 \times 10^{-11}$ \\
D & $1.1 \times 10^{-5}$ & $1.5 \times 10^{-4}$ & $1.5 \times 10^{-11}$ \\
E & $2.4 \times 10^{-7}$ & $7.9 \times 10^{-9}$ & $1.0 \times 10^{-14}$ \\
F & $4.7 \times 10^{-4}$ & $2.7 \times 10^{-3}$ & $1.0 \times 10^{-9}$ \\
G & $3.8 \times 10^{-3}$ & $2.6 \times 10^{-2}$ & $1.0 \times 10^{-7}$ \\
H & $8.3 \times 10^{-3}$ & $1.3 \times 10^{-2}$ & $2.4 \times 10^{-5}$ \\
\hline
\end{tabular}

Table 2. For each $y$-map B-H, we list the probability that the fit in Eq. (5.1) allows: $\alpha=0, \gamma=1$ (no 1-halo term, column 2); $\alpha=1, \gamma=0$ (no 2-halo term, column 3); and $\alpha=\gamma=0$ (no cross-correlation, column 4). We assume $P=\exp \left(-\Delta \chi^{2} / 2\right)$.

$33 \%, 44 \%$, and $32 \%$, respectively. In the right panel, we sample the entire cosmological parameter space by using the posterior sample chains released for WMAP 9-year $\Lambda$ CDM fits and the Planck 2015 "plik HM TTTEEE lowTEB" chain, and plot the predicted uncertainties for the UP model by using the software COSMOSIS [44]. The $68 \%$ and $95 \%$ band for $\xi^{\kappa y}$ are shown in deep and shallow colours, respectively. One can see that, the CL bands of $\xi^{\kappa y}$ for WMAP are much larger than for Planck, due to its larger parameter uncertainties. However, since Planck results have smaller uncertainties, in the following we will use the Planck cosmological parameters. We therefore remind the reader that the subsequent conclusions are based on the Planck cosmological parameters.

\section{Constraining the baryon component with the $\beta$-model}

To study the $\beta$-model further, we separately examine the 1-halo and 2-halo contributions, as shown in Fig. 4. The 2-halo term captures the effects of halo clustering, so it produces a flatter contribution to $\xi^{\kappa y}(\theta)$ than the 1-halo term. To some extent, this term is a proxy for gas at large radii, not captured by the pressure profile in the 1-halo term. Any tendency for the data to favour a higher-than-predicted 2-halo contribution might be pointing to the need for additional diffuse gas. As expected, the 1-halo term dominates at small scales, while the 2-halo term dominates at large scales, with a crossover point at $13 \mathrm{arcmin}$. For an average lens at $z \simeq 0.37$, this corresponds to a physical length of $4 \mathrm{Mpc}$. We quantify the relative contributions of the two terms by fitting each with scaling coefficients $\alpha$ and $\gamma$ :

$$
\chi^{2}(\alpha, \gamma)=\sum_{i j}\left[\xi^{\mathrm{d}}\left(\theta_{i}\right)-\alpha \xi^{1 \mathrm{~h}}\left(\theta_{i}\right)-\gamma \xi^{2 \mathrm{~h}}\left(\theta_{i}\right)\right] C_{i j}^{-1}\left[\xi^{\mathrm{d}}\left(\theta_{j}\right)-\alpha \xi^{1 \mathrm{~h}}\left(\theta_{j}\right)-\gamma \xi^{2 \mathrm{~h}}\left(\theta_{j}\right)\right] .
$$

Fig. 5 shows the constraints on $(\alpha, \gamma)$ for the Planck-CFHTLenS cross-correlation using data set D (although the other $y$-maps give similar results). Even though the nominal model, $(\alpha, \gamma)=(1,1)$, is within the $95 \%$ contour, the data prefer a fit with somewhat higher 2-halo amplitude compared with 1-halo, which we interpret as an indication that the $\kappa-y$ cross-correlation favours gas that is further out in halos. Models with no correlation, $(\alpha, \gamma)=(0,0)$, or with only 1-halo or 2-halo contributions, $(\alpha, \gamma)=(1,0)$ or $(0,1)$, respectively, are strongly rejected. We quantify this for data sets B-H in Table 2, by evaluating $\Delta \chi^{2}=\chi^{2}(\alpha, \gamma)-\chi_{\min }^{2}$, where $\chi_{\min }^{2}$ corresponds to the best-fit $(\alpha, \gamma)$. We measure the contributions from $1-$ and 2 -halo terms by calculating the fractional area under the correlation function, i.e., $\int \xi^{\kappa y, 1 \mathrm{~h} / 2 \mathrm{~h}}(\theta) \mathrm{d} \theta / \int \xi^{\kappa y}(\theta) \mathrm{d} \theta$, finding that they each contribute about $50 \%$ of the signal.

To further probe contributions from gas at large radii and in low-mass halos, we segregate the integrals in the $\beta$-model by mass and radius. First, we truncate the gas distribution at one virial radius 


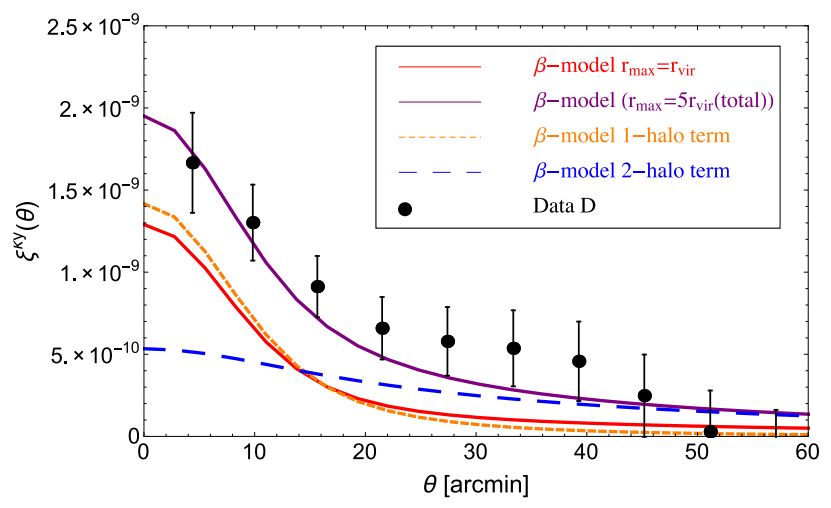

Figure 4. Separate contributions to the halo model using the $\beta$-model pressure profile. The dashed lines show the contributions from the 1-halo (orange) and 2-halo (blue) terms; the solid lines show the effect of truncating the pressure profile integral in Eq. (3.3) at different radii.

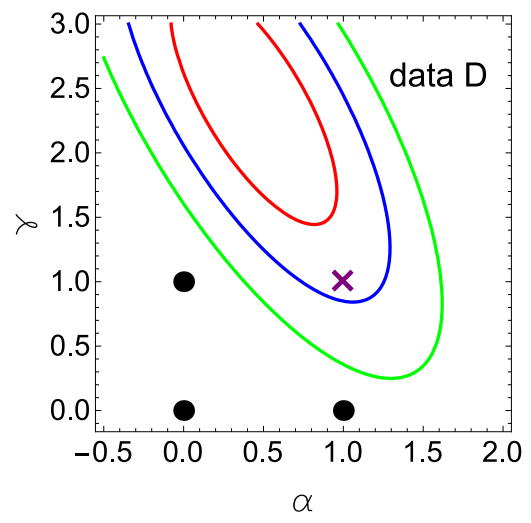

Figure 5. Joint constraints on $\alpha$ and $\gamma$ from Eq. (5.1) for specific data set D (other choices are not dramatically different), showing the $68.3 \%, 95.4 \%$ and $99.7 \%$ confidence contours. The model correlation function used here is based on the isothermal $\beta$-model, and the nominal model $(\alpha=\gamma=1)$ is indicated by the purple cross. The black dots are for models with no correlation $(\alpha=\gamma=0)$ and with separate 1-halo and 2-halo terms.

in Eq. (3.3): $x_{\max }=a(z) r_{\mathrm{vir}} / r_{\mathrm{s}}$, as shown in Fig. 4. Next, we divide the model contributions into two mass bins, $10^{12}-10^{14} \mathrm{M}_{\odot}$ and $10^{14}-10^{16} \mathrm{M}_{\odot}$, and two radial bins, $r \leq r_{\mathrm{vir}}$ and $r \geq r_{\mathrm{vir}}$. The fractional contributions to the integrated signal are presented in Table 3. Given our model assumptions, nearly half of the integrated signal $(46 \%)$ originates from baryons outside the virial radius of dark matter halos, while $40 \%$ originates from low-mass halos. One can additionally calculate the fraction of baryons found inside the virial radius, $f=\int_{0}^{r_{\mathrm{vir}}} n_{\mathrm{e}}(r) r^{2} \mathrm{~d} r / \int_{0}^{\infty} n_{\mathrm{e}}(r) r^{2} \mathrm{~d} r$. This $f$ function is redshift and mass dependent, but if we take the median value of the redshift distribution $z=0.37$ and median mass $10^{14} \mathrm{M}_{\odot}$ into the above expression, we find $f=35 \%$, meaning that $65 \%$ of the baryons are, on average, located beyond the virial radius.

One point to recall about our $\beta$-model predictions is that we assume the gas to be isothermal, with temperature calibrated against 24 hydrodynamic cluster simulations [31]. Although this assumption is less likely to hold for gas outside the virial radius, Ref. [31] shows that this single temperature model produces an excellent fit to photon spectra.If the outer gas is cooler than the inner gas, we would have to boost the gas density to retain the $\kappa-y$ signal we observe, and vice-versa. Note that, for halos at redshift $z=0.37$ (the mean probed by the CFHTLens sample), the effective temperature 


\begin{tabular}{c|cc} 
& $10^{12} \mathrm{M}_{\odot}-10^{14} \mathrm{M}_{\odot}$ & $10^{14} \mathrm{M}_{\odot}-10^{16} \mathrm{M}_{\odot}$ \\
\hline$(0.01-1) r_{\text {vir }}$ & $26 \%$ & $28 \%$ \\
$(1-100) r_{\text {vir }}$ & $14 \%$ & $32 \%$
\end{tabular}

Table 3. Fractional contribution to the model cross-correlation function arising from different mass and radial profile cuts.
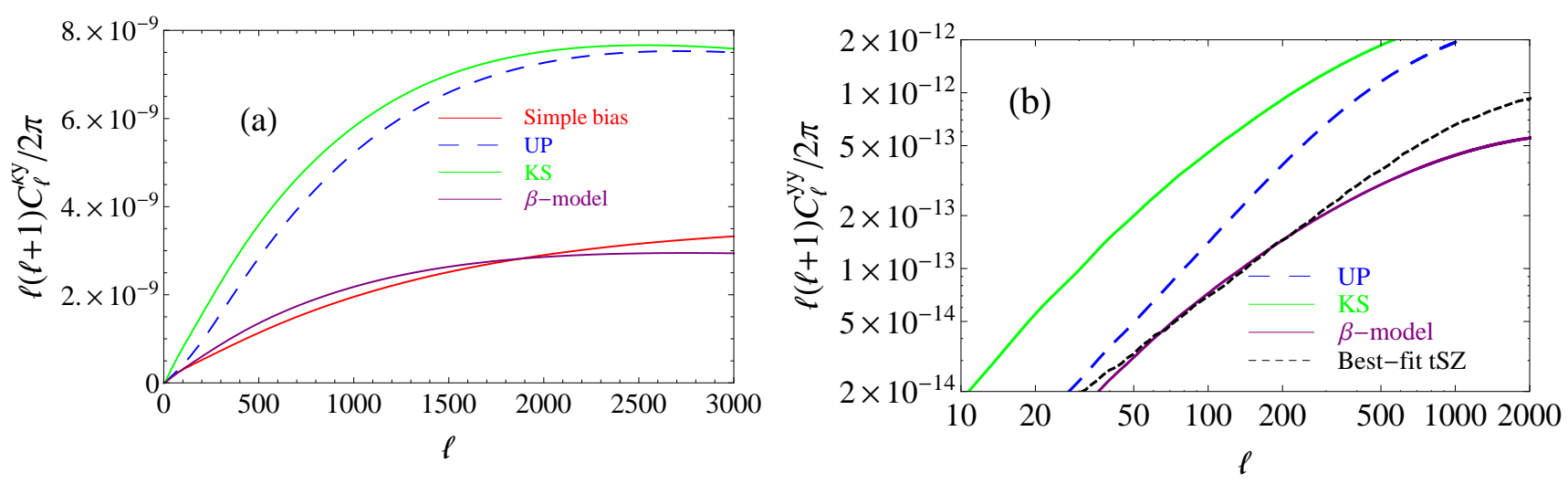

Figure 6. Predictions of the halo model for (a) $C_{\ell}^{\kappa y}$ and (b) $C_{\ell}^{y y}$. The prediction for the auto-correlation, $C_{\ell}^{y y}$, using the $\beta$-model profile, agrees reasonably well with the best-fit tSZ spectrum measured by Planck [19] (dashed line).

ranges between $T_{\mathrm{vir}} \simeq 7 \times 10^{5} \mathrm{~K}$ and $3 \times 10^{8} \mathrm{~K}$ for halos in the range $10^{12}-10^{16} \mathrm{M}_{\odot}$ (equation (14) in [26]). The lower end of this range agrees with the expected temperature of the warm phase of the intergalactic medium residing in filaments and sheets of clustered matter. Thus it is plausible that our measurement is, in fact, probing warm gas associated with lower mass halos that could constitute the missing baryons.

Our analysis of the cross-correlation signal can also be used to predict the tSZ power spectrum $C_{\ell}^{y y}$, which can then be compared to the measurement made by the Planck team [19]. Replacing $\kappa_{\ell}$ by $y_{\ell}$ in Eqs. (3.1) and (3.6), and using the $\beta$-model for the pressure profile, we show our predicted $C_{\ell}^{y y}$ in Fig. 6b. The agreement with the power spectrum derived directly from the Planck maps is quite good, while the predictions based on the KS and UP profiles are clearly too high. Note that our prediction is only correct if the correlation coefficient $r$ between the 3-d pressure and matter distributions is 1 . Hydrodynamical simulations in Ref. [46] find $r \sim 0.5$, but this conclusion is still uncertain, so our prediction should only be regarded as a lower limit.

\section{Discussion and Conclusions}

Our halo model for the lensing-tSZ cross-correlation signal $\xi^{\kappa y}$ has enabled us to investigate the baryon distribution at cluster scales and to explore the possible identification of the missing baryons in the warm-hot intergalactic medium (WHIM). The observed cross-correlation function from the CFHTLenS mass map and the Planck tSZ map is particularly effective at tracing baryons over a wide range of clustering scales.

In the context of the universal pressure profile, we find that their predicted $\xi^{\kappa-y}(\theta)$ function is higher than the observational data at small angular scales; the added hydrostatic mass bias $(1-b) \simeq$ 0.8 can reconcile the tension to some extent, but on large angular scales it predicts lower power than 
seen observationally. By employing a likelihood function to fit the $(1-b)$, we find its value to be $(1-b)=0.79_{-0.1}^{+0.07}$ (at $95 \% \mathrm{CL}$ ), which is consistent with previous values found values in numerical simulations [38-42], as well as some observational constraints [33, 34, 36].

In the context of the isothermal $\beta$ profile, the 1- and 2-halo terms are each detected at $\sim 4 \sigma$, while the total signal is detected at $\sim 6 \sigma$. We find evidence that baryons are distributed beyond the virial radius, with a temperature in the range of $\left(10^{5}-10^{7}\right) \mathrm{K}$, consistent with the hypothesis that this signal arises from the missing baryons. We further separate the model signal into different radial profile and mass bins, and find that about half of the integrated signal arises from gas outside the virial radius of the dark matter halos, and that $40 \%$ arises from low-mass halos.

Our study is an example of a general class of large-scale cross-correlations that are now becoming feasible, thanks to the availability of deep multi-waveband surveys over large fractions of the sky. Correlation of tSZ maps with galaxies [47], with CMB lensing [37] and with X-rays [48], plus the use of correlations with the kinetic SZ effect [49, 50], allow for a multi-faceted study of the role of baryon physics in structure formation. Further improvements in the quality of the data will require more sophisticated models than we have presented here, perhaps involving direct comparison of diagnostics of the WHIM with hydrodynamical simulations. Our results show that such cross-correlation studies have the potential to trace the "missing baryons" and to account for the cosmic baryon distribution with high precision.

\section{Acknowledgments}

We would like to thank Anna Bonaldi, J. Colin Hill, Houjun Mo, Pengjie Zhang, and Eiichiro Komatsu for helpful discussions. This paper made use of the Planck Legacy Archive (http: / / archives . esac . esa and the Canada-France-Hawaii Telescope Lensing Survey (http://www. cfhtlens.org/).

\section{References}

[1] A. J. Benson, 2013, PhR, 495, 33

[2] J. Tinker et al., 2013, ApJ, 778, 93

[3] M. Fukugita, and P. J. E. Peebles, 2004, ApJ, 616, 643

[4] R.A. Sunyaev, and Y. B. Zeldovich, 1972, CoASP, 4, 173

[5] J. N. Bregman, 2007, ARAA, 45, 221

[6] R. Cen, and J. Ostriker, 2006, ApJ, 650, 560

[7] L. van Waerbeke, G. Hinshaw, \& N. Murray, 2014, PRD, 89, 023508

[8] A. Cooray, \& R. Sheth, 2002, Phys. Rep. 372, 1

[9] Planck Collaboration 2013 XVI, A\&A, 571, 16

[10] J. Benjamin et al., 2013, MNRAS, 431, 1547

[11] C. Heymans et al., 2012, MNRAS, 427, 146

[12] H. Hildebrandt et al., 2012, MNRAS, 421, 2355

[13] T. Erben et al., 2013, MNRAS, 433, 2545

[14] L. Miller et al., 2013, MNRAS, 429, 2858

[15] Planck Collaboration, Planck 2013 results. XVI, 2014, A\&A, 571, 1

[16] Planck Collaboration, Planck Intermediate Results. XXII, 2015, A\&A, 576, 107 
[17] R. K. Sheth, and G. Tormen, 2002, MNRAS, 329, 61

[18] J. F. Navarro, C. S. Frenk, and S. D. M. White, 1996, ApJ, 462, 563

[19] Planck Collaboration 2013 XVI, A\&A, 571, 21

[20] A. R. Duffy, J. Schaye, S. T. Kay, \& C. Dalla Vecchia, 2008, MNRAS, 390, L64

[21] A. Lewis, A. Challinor, and A. Lasenby, 2000, ApJ, 538, 473; http: / / www . camb . info

[22] H. Mo, and S. D. M. White, 2002, MNRAS, 336, 112

[23] E. Komatsu, and U. Seljak, 2002, MNRAS, 336, 1256

[24] M. Arnaud et al., 2010, A\&A, 517, 92

[25] M. Arnaud, 2009, A\&A, 500, 103

[26] J.-C. Waizmann, and M. Bartelmann, 2009, A\&A, 493, 859

[27] E. J. Hallman, J O. Burn, P. M. Motl, \& M. L. Norman, 2007, ApJ, 665, 911

[28] T. Plagge et al., 2010, ApJ, 716, 1118

[29] E. Komatsu et al., 2011, ApJS, 192, 18

[30] Planck Collaboration, Planck Intermediate Results V., 2013, A\&A, 550, 131

[31] B. F. Mathiesen, \& A. E. Evrard, 2001, ApJ, 546, 100

[32] A. von der Linden, et al., 2014, MNRAS, 443, 1973

[33] H. Hoekstra, et al., 2015, MNRAS, 449, 685

[34] H. Israel et al., 2014, A\&A, 564, 129

[35] M. Simet, N. Battaglia, R. Mandelbaum, \& U. Seljak, 2015, arXiv:1502.01024

[36] Planck Collaboration, Planck 2015 Results XXIV., 2015, arXiv: 1502.01597

[37] J. C. Hill, and D. Spergel, 2014, JCAP, 02, 030

[38] L. D. Shaw, D.Nagai, S. Bhattacharya, E. T. Lau, 2010, ApJ, 725, 1452

[39] E. Rasia et al., 2012, New Journal of Physics, 14, 055018

[40] D. Nagai, A. V. Kravtsov, \& A. Vikhlinin, 2007, ApJ, 668, 1

[41] R. Piffaretti, \& R. Valdarnini, 2008, A\&A, 491, 71

[42] M. Meneghetti, et al. 2010, A\&A 514, A93

[43] J-B. Melin, \& J. G. Bartlett, 2015, A\&A, 578, 21

[44] J. Zuntz, et al., 2015, Astronomy and Computing, 12, 45

[45] E. Pierpaoli, D. Scott, and M. White, 2001, MNRAS, 325, 77

[46] U. Seljak, J. Burwell, \& U. L. Pen, 2001, PRD, 63, 063001

[47] Planck Collaboration, 2013, A\&A, 557, A52

[48] A. Hajian, et al., 2013, JCAP, 11, 064

[49] N. Hand, et al., 2012, PRL, 109, 041101

[50] M. Li, R. E. Angulo, S. D. M. White, \& J. Jasche, 2014, MNRAS, 443, 2311 